\title{
A New Iris Recognition Approach Based on a Functional Representation
}

\author{
Dania Porro-Muñoz, Francisco José Silva-Mata, Victor Mendiola-Lau, \\ Noslen Hernández, and Isneri Talavera \\ Advanced Technologies Application Center (CENATAV) - Cuba \\ \{dpmunoz,fjsilva,vmendiola,nhernandez,i talavera\}@cenatav.co.cu
}

\begin{abstract}
This paper proposes the introduction of annular Zernike polynomials for representing iris images data. This representation offers notables advantages like representing the images on a continuous domain that allows the application of Functional Data Analysis techniques, preserving their original nature. In addition, it provides a significant dimensionality reduction of the data, while it still has a high discriminative power. The proposed approach also deals with the occlusion problems that can be present in this type of images. In order to corroborate the effectiveness of the introduced approach, identification experiments were carried out. Iris international databases were used. Some of them are characterized by the presence of severe occlusion problems. Results have shown high recognition accuracy.
\end{abstract}

Keywords: Iris recognition, Functional Data Analysis.

\section{Introduction}

Eyes texture has practically unrepeatable patterns among human beings, even twins, which makes the iris a biometric entity usable for people identification [1]. In iris recognition research area, there are still many open problems that require of innovative solutions related to the steps of iris recognition(image capture, eye localization, segmentation, noise detection, normalization, feature extraction and matching) [2].

Traditionally, iris features have been represented by high-dimensional vectors. However, the fact that digital images data are recorded discretely, though their nature is continuous is ignored. Therefore, instead of representing these images by vectors, it might be more appropriate to represent the iris image data by an underlying continuous function, in a way that this representation can be closer to the original nature of the data. Using functions for these images representation brings many advantages, such as: data is represented as a whole, the revelation of the dynamic aspects of the original data, the ability of analyzing some of the most significant features of the function, for example, the monotony, differentiability and smoothness, and a substantial dimensionality reduction of the data[3]. An image can be represented as a function $f(x, y)$ over 
a spatial domain [4]. Finding such function that better approximates the discrete observed data is one of the key points of this approach. Using functional basis expansion is a common issue for this representation. The selection of the basis set and the estimation of the appropriate number of coefficients must be performed thinking in obtaining a sufficiently discriminative representation with a minimum dimension. The proposed solution for the particular case of iris images, demonstrates the validity of the approach and traces a new general methodology for analyzing biometric images.

The paper is organized as follows. In Section 2 the iris recognition process is explained, and our introduced approach is detailed. In Section 3 the performed experiments and results are described. Conclusions and future research topics are drawn in Section 4.

\section{Iris Recognition Process}

Iris recognition process consists on the general steps(image capture, eye localization, segmentation, noise detection, normalization, feature extraction and matching). Nevertheless, these steps present some peculiarities when Functional Data Analysis(FDA) approach is used. The general diagram presented in Fig. 1 shows the necessary steps to perform an image recognition task based on functional data analysis. This helps for a better understanding of the process.

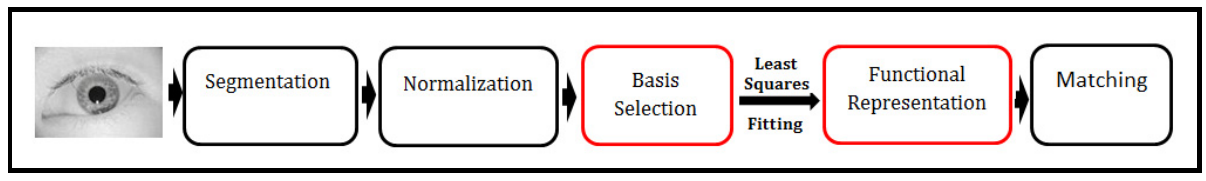

Fig. 1. A general description of the iris image analysis using FDA

\subsection{Segmentation and Normalization}

The segmentation step is applied to separate the iris region from the other part of the eye image. The main goal of this step is to extract the region of interest(ROI), containing the maximum amount of pixels with valid information, and the minimum with irrelevant information. During the normalization stage, the isolated region of the iris is reduced to specific dimensions(specified by the radial and angular resolution) using the coordinates transformation.

For these two steps of the iris recognition process(segmentation and normalization), we used the third module(Iris recognition) of the known Video-based Automated System for Iris Recognition (VASIR) [5]. This system segments the iris region using their own segmentation approach. Those regions are then extracted and normalized based on the known "rubber-sheet" method. Each point within the iris region is assigned a pair of real coordinates $(r, \theta)$ contained in 
a rectangle where the radius $r$ lies on the unit interval $[0,1]$ and $\theta$ is the angle over $[0,2 \Pi]$.

Before constructing the functional representation, it must be ensured that it is the least affected by the factors that can be present in the ROI. This factors could be: dilation, specular reflection, iris resolution, motion blur, camera diffusion, presence of eyelids, eyelashes, and others [6]. In particular, the occlusion by eyelashes and eyelids significantly affects the behavior of this type of representation.

Some solutions use binary masks to face these occlusion problems [7]. However, most of them are based on representations that use local features. Other solutions have restricted the region of interest of the iris domain[8], by taking into account its statistical behavior with respect to the presence of occlusions. This region should be the least affected by occlusions of eyelids and eyelashes. In our research, we built our representation after selecting different regions of the iris images, as explained below. In Fig.2(a), half of the iris region was re-

a)

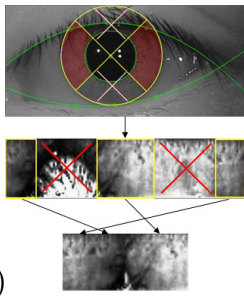

b)
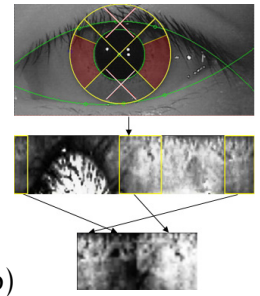

c)

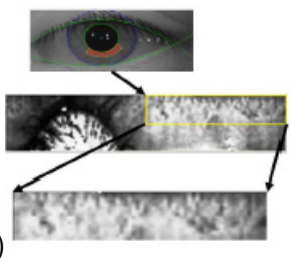

Fig. 2. Three different regions with different of occlusion levels

moved, keeping the region comprised in $[-\pi / 4,+\pi / 4]$ and $[3 \pi / 4,5 \pi / 4]$ (upper and bottom sectors indicated with a cross ) [7]. In Fig.2(b) it is shown that those sectors selected in Fig,2 a) were reduced, in order to decrease the incidence of the eyelids and eyelashes comprised in $[-\pi / 4,+\pi / 8]$ and $[7 \pi / 8,5 \pi / 4]$. Finally, in Fig 2 c), a $1 / 4$ of the rectangular region is selected, minimizing even more the incidence of occlusion as is proposed in [8]. For each of the selected regions, all the images were reduced to an specific dimension $32 \times 128,32 \times 48$ and $16^{*} 64$ respectively.

\subsection{Representation}

After the image has been normalized, we proceed to the construction of the functional representation. The method consists in approximating(smoothing) each image by a linear combination (weighted sum) of basis functions, which is a common strategy for achieving this type of representation [3].

$$
I_{f}(x, y)=\sum_{k=1}^{K} c_{k} b_{k}(x, y),
$$


where $\left\{b_{k}(x, y)\right\}_{k}$ denotes the set of $k$ bases functions and $\left\{c_{k}\right\}_{k}$ represents the coefficients of the expansion.

Finally, from the discrete observations $I(x, y)$ i.e, the iris normalized images, a functional representation $\hat{I}_{f}(x, y)$, is obtained. Images will now be described by the coefficients $\left\{c_{k}\right\}$. It has been demonstrated that working with these coefficients is strictly equivalent to working directly on the $b_{k}$ functions [9].

One important step to consider in this process is to choose the best basis set to expand the function. There are several criteria for selecting the basis set, which are explained bellow.

Basis Selection and Determination of the Number of Coefficients. Some of the principal criteria that are considered for basis selection are: the geometry of the domain [10], computational complexity, differentiability, periodicity of the event to model, the ratio of speed of convergence, completeness (understood as the ability to represent any function with high precision and with enough terms coefficients). For our selection, we will mainly focus on the geometry of the domain. Since we are interested in approximating functions of two variables, we need bivariate basis functions for the expansion. The annular Zernike polynomials are a basis functions set defined over an unitary annulus, which is suitable for modeling the iris domain due to its shape.

Any function $I_{f}(\rho, \theta)$ defined over a two-dimensional space can be approximated using the annular Zernike polynomials bases[11]. Thus, the equation for expanding a sector of an annular iris region $\left(\hat{I}_{f}(\rho, \theta, \epsilon)\right)$ in terms of annular polynomials $Z_{n}^{m}(\rho, \theta, \epsilon)$ that are orthonormal over a unit annulus is:

$$
\hat{I}_{f}(\rho, \theta, \epsilon)=\sum_{n}^{\infty} \sum_{m}^{n} C_{n}^{m} Z_{n}^{m}(\rho, \theta, \epsilon)
$$

where $C_{n}^{m}$ represents the vector of coefficients and $Z_{n}^{m}$ represents the annular Zernike polynomials basis functions. The annular Zernike polynomials are similar to circular Zernike polynomials, except that are orthonormal in an annulus instead of a circle [11]. They are usually defined in polar coordinates $(\rho, \theta)$. The parameter $\rho \in[0,1]$, is the radial coordinate, and $\theta \in[0,2 \pi]$, the azimuthal component. Annular Zernike polynomials have inner radius $\epsilon$ and outer radius 1 , and thus the coordinate $\rho$ is subject to the restriction $0 \leq \epsilon \leq 1$. For the specific case of iris, the value $\rho$ is comprised between the pupillary boundary and the limbus boundary.

These polynomials are derived from the circular Zernike polynomials by Gram-Schmidt orthogonalization process[11]. Each Zernike polynomial is a tensor product of Fourier bases in the angular direction and a special type of Jacobi polynomials in the radial direction[11]. It consists of 3 components: a normalization factor, a radial component and one azimuthal component. Annular Zernike polynomials are then defined as follows:

$$
Z_{n}^{ \pm m}=\left\{\begin{array}{lll}
N_{n}^{m} R_{n}^{|m|}(\rho, \epsilon) \cos (m \theta) & \text { if } & m \geq 0 \\
-N_{n}^{m} R_{n}^{|m|}(\rho, \epsilon) \sin (m \theta) & \text { if } & m<0 .
\end{array}\right.
$$


For a given radial order or polynomial order $n$, the azimuthal frequency or Fourier order $m$ can only take values of $-n,-n+2,-n+4, \ldots, n[11] . N_{n}^{m}$ is the normalization factor

$$
N_{n}^{m}=\sqrt{\frac{2(n+1)}{1+\delta_{m 0}}}, \quad \text { with } \quad \delta_{m 0}=\left\{\begin{array}{lll}
1 & \text { if } & m=0 \\
0 & \text { if } & m \neq 0
\end{array},\right.
$$

and $R_{n}^{|m|}(\rho, \epsilon)$ is the representation for the Jacobi polynomial, which for the general case can be expressed as:

$$
\mathrm{R}_{2 j+m}^{m}(\rho, \epsilon)=\left[\frac{1-\epsilon^{2}}{2(2 j+m+1) \mathrm{h}_{j}^{m}}\right]^{\frac{1}{2}} \rho^{m} \mathrm{Q}_{j}^{m}\left(\rho^{2}\right)
$$

where $\left\{Q_{j}^{m}(u)\right\} \sqrt[1]{1}$ is a set of orthogonal polynomials obtained by the orthonormalization of the sequence $1, u, \ldots, u^{j}$ over the interval $\left(\epsilon^{2}, 1\right)$. It is defined as:

$$
Q_{j}^{m}(u)= \begin{cases}R_{2 j}^{0}(\rho, \epsilon) & \text { si } m=0 \\ \frac{2(2 j+2 m-1)}{(j+m)\left(1-\epsilon^{2}\right)} \frac{h_{j}^{m-1}}{Q_{j}^{m-1}(0)} \sum_{i=0}^{j} \frac{Q_{i}^{m-1}(0) Q_{i}^{m-1}(u)}{h_{i}^{m-1}} \text { e.o.c }\end{cases}
$$

These expressions were taken from [11]. In order to obtain an estimate $\hat{I}_{f}(\rho, \theta)$ of $I_{f}(\rho, \theta)$ for each image $I(\rho, \theta)$, we need to estimate the coefficients in the expansion. This will be done by least squares fitting. Due to the fact that Zernike polynomials are orthonormal in the unit sphere, i.e.,

$$
\frac{1}{\pi\left(1-\epsilon^{2}\right)} \int_{\epsilon}^{1} \int_{0}^{2 \pi} Z_{n}^{m}(\rho, \theta, \epsilon) Z_{n^{\prime}}^{m^{\prime}}(\rho, \theta, \epsilon) \rho d \rho d \theta=\delta_{n n^{\prime}} \delta_{m m^{\prime}}
$$

the operations (e.g., inner products and norms) between functions expressed on this basis, get reduced to operations between their corresponding coefficients, which makes the computations easier. Thus, as it was explained at the beginning of this section, every analyzed image will be represented by the coefficients obtained from the linear combination of the annular Zernike polynomials, expressed in Eq 2. There is still one hyperparameter that needs to be established before doing the least square estimation of the coefficients, and it is the number of coefficients (or number of basis functions) in the expansion. This parameter has the role of a smoothing parameter. Statistically, keeping a few coefficients in the expansion is equivalent to conducting heavy amount of smoothing for the original data. It also determines the dimensionality reduction achieved with this representation. One way for determining this parameter is through the bootstrapping strategy [12].

${ }^{1}$ Note that $u=\rho^{2}$. 


\section{Experimental Results}

To evaluate the performance of the proposed method, we used the CASIAV2[13], UPOL[14], and MMU[15] databases. CASIA database version 2.0 consists of 1200 iris images from 60 different irises (subjects) with a resolution of 640 x 480 pixels. The MMU v1.0 database contains 450 iris images, which were collected from 45 subjects. There are 10 images from each subject. These are images of $320 \times 240$ pixels. This database has images with problems like specular reflections, off-axis and off-angle, blur, focus, non-uniform illumination, occlusions such as eyelids, eyelashes, glasses, contact lens, and hair.The UPOL database contains 384 images of 576 x 768, extracted from both eyes of 64 subjects (three images per eye). In our experiments, we used the VASIR method for the segmentation and normalization as we explained in Sec.1. Once we have images normalized, including the selection of the region avoiding the occlusions(explained in Sec.2), we represent them by using FDA, like is explained below: a) In order to obtain the optimal number of coefficients for the functional representation, we split the data sets in training ( $80 \%$ of the data) and test $(20 \%)$. On each training set, a bootstrap method was then applied to find this optimal number. Results are then given by evaluating the test sets with the selected number of coefficients. It should be noted, that for the three cases of the selection of the region, all the resultant subregions have the same size. Taking this into account, the bootstrap process was applied to the UPOL database, and the optimal number obtained ( 48 coefficients) was used for all the databases.

$b$ ) The coefficients were estimated by the least squares fitting method on the annular Zernike polynomial basis using the normalized image. This process was repeated for the three different regions of interest selected with different affectations of occlusion.

c) The step $b$ ) was applied for every image in the databases, and then performed iris recognition by using an Euclidean distance between the coefficients. The recognition results were compared with Daugman[1] and Masek[16] representations. In Tables 1, 2 and 3, we show the results of the identification experiments with the images represented by the optimal number of coefficients (48) for each of the selected regions shown in Fig 2 In order to corroborate the importance of obtaining the optimal number of coefficients for a good representation, some experiments were carried out using 16 and 32 coefficients too. As it can be seen, the best results for the 3 databases, were obtained in Table 3

Table 1. Recognition results on CASIA V2, MMU V1 and UPOL databases, with region of interest represented in Fig 2 a)

\begin{tabular}{|c|c|c|c|c|c|}
\hline Database & $\mathbf{1 6}$ & $\mathbf{3 2}$ & $\mathbf{4 8}$ & Daugman & Masek \\
\hline CASIA2 & 95.33 & 96.75 & 97.42 & 98.75 & 98.00 \\
\hline MMU1 & 94.22 & 95.56 & 96.22 & 97.33 & 97.56 \\
\hline UPOL & 97.22 & 98.96 & 99.74 & 100 & 100 \\
\hline
\end{tabular}


Table 2. Recognition results on CASIA V2, MMU V1 and UPOL databases, with region of interest represented in Fig 2 b)

\begin{tabular}{|c|c|c|c|c|c|}
\hline Database & $\mathbf{1 6}$ & $\mathbf{3 2}$ & $\mathbf{4 8}$ & Daugman & Masek \\
\hline MMU1 & 94.67 & 96.89 & 97.11 & 97.33 & 97.56 \\
\hline CASIA2 & 96.83 & 98.25 & $\mathbf{9 8 . 7 5}$ & 98.75 & 98.00 \\
\hline UPOL & 99.22 & 99.48 & 99.74 & 100 & 100 \\
\hline
\end{tabular}

Table 3. Recognition results on CASIA V2, MMU V1 and UPOL databases, with region of interest represented in Fig 2 c)

\begin{tabular}{|c|c|c|c|c|c|}
\hline Database & $\mathbf{1 6}$ & $\mathbf{3 2}$ & $\mathbf{4 8}$ & Daugman & Masek \\
\hline CASIA2 & 98 & $\mathbf{9 9 . 2 5}$ & $\mathbf{9 9 . 7 5}$ & 98.75 & 98.00 \\
\hline MMU1 & 93.56 & 96.85 & 97.11 & 97.33 & 97.56 \\
\hline UPOL & 99.22 & 99.48 & 99.74 & 100 & 100 \\
\hline
\end{tabular}

(highlighted with black letter). In this case, the upper-right region of the image was used, according to the Fig.2(c), and then represented with the optimal number of coefficients. It should be noted that in this case our method overcomes the results from Daugman and Libor Masek in the Casia2 database(database with severe occlusions), while we achieved a dimensionality reduction of a $50 \%$ and $25 \%$ with respect to Daugman and Masek respectively. In the case of MMU1 and UPOL, although the results did not overcome the other methods, the achieved values of accuracy were high. The results from Table 2, representing the images that correspond to the region mentioned in Fig.2(b), are comparable to those of other methods, but it must still be highlighted the low dimensionality of our representation. Finally, analyzing the results from Table 1, it can be seen that occlusion factors affected slightly the accuracy results, but they are good overall. In general, it can be seen that our approach obtained good results with representations of very low dimensionality. It should also be noted that the best results were obtained with the representation constructed from the upper-right region of the iris image (Fig.2(c)), which is the less affected by occlusions. This shows somehow the sensitivity of the proposed representation to the occlusion problems. Therefore, the need of dealing with this problem (in this case by selecting the significative regions of the images) before obtaining the functional representation of the iris. In the 3 experiments, with the different selections of regions, the best accuracy was obtained with the optimal number of coefficients, mainly for those images where occlusion is present. This shows the importance of finding an optimal number of coefficients, for a good performance of our method.

\section{Conclusions and Future Works}

In this paper, we present a new functional iris representation based on annular Zernike polynomials. The main contributions of this work are: a high 
recognition accuracy in presence of severe occlusion problems while the dimensionality of the data is significantly reduced. It was demonstrated that with the proposed representation a high recognition rate can be obtained by just analyzing a small subregion of the iris image. For future work we should perform more experiments with other iris images databases to corroborate the performance of our method. Moreover, we are planning to extend this approach to other biometric images.

\section{References}

[1] Daugman, J.: How iris recognition works. IEEE Trans. CSVT 14(1), 21-30 (2004)

[2] Jan, F., Usman, I., Agha, S.: Iris localization in frontal eye images for less constrained iris recognition systems. Digital Signal Processing 22, 971-986 (2012)

[3] Ramsay, J.O., Silverman, B.W.: Functional Data Analysis, 2nd edn. Springer, Heidelberg (2005)

[4] Gonzalez, R.C., Woods, R.E.: Digital Image Processing, 2nd edn. Prentice Hall Upper Saddle River, New Jersey 07458 (2001)

[5] Lee, Y., Phillips, P.J., Micheals, R.J.: An automated video-based system for iris recognition. In: Tistarelli, M., Nixon, M.S. (eds.) ICB 2009. LNCS, vol. 5558, pp. 1160-1169. Springer, Heidelberg (2009)

[6] Chaskar, U.M., Sutaone, M.S., Shah, N.S., Jaison, T.: Iris image quality assessment for biometric application. IJCSI International Journal of Computer Science Issues 9(3), 474-479 (2012)

[7] Vatsa, M., Singh, R., Noore, A.: Improving iris recognition performance using segmentation, quality enhancement, match score fusion, and indexing. IEEE Transactions on Systems, Man, and Cybernetics, Part B: Cybernetics 38(4), 1021-1035 (2008)

[8] Lin, Z., Lu, B.: Iris recognition method based on the optimized gabor filters. In: 3rd International Congress on Image and Signal Processing (CISP 2010) (2010)

[9] Rossi, F., Delannay, N., Conan-Guez, B., Verleysen, M.: Representation of functional data in neural networks. Neurocomputing 64(2), 183-210 (2005)

[10] Boyd, J.P.: Chebyshev and Fourier Spectral Methods: Second Revised Edn. Springer (1989)

[11] Mahajan, V.N., Aftab, M.: Systematic comparison of the use of annular and zernike circle polynomials for annular wavefronts. Appl. Opt. 49(33), 6489-6501 (2010)

[12] Iskander, D.R., Collins, M.J., Davis, B.: Optimal modeling of corneal surfaces with zernike polynomials. IEEE Transactions on biomedical engineering 48, 8795 (2001)

[13] Institute of Automation, Chinese Academy of Sciences, CASIA Iris Image Database (2004), http://www.sinobiometrics.com

[14] Dobes and Libor Machala.Upol iris image database (2004), http://phoenix.inf.upol.cz/iris

[15] Multimedia University, MMU Iris Image Database. (2004), http://pesona.mmu.edu.my/ccteo

[16] Masek, L.: Recognition of human iris pattern for biometric identification. Master's thesis, School of Computer Science and Software Engineering, The University of Western Australia (2003) 